\title{
Visible and near infrared radiation may be transmitted or absorbed differently by beetle elytra according to habitat preference
}

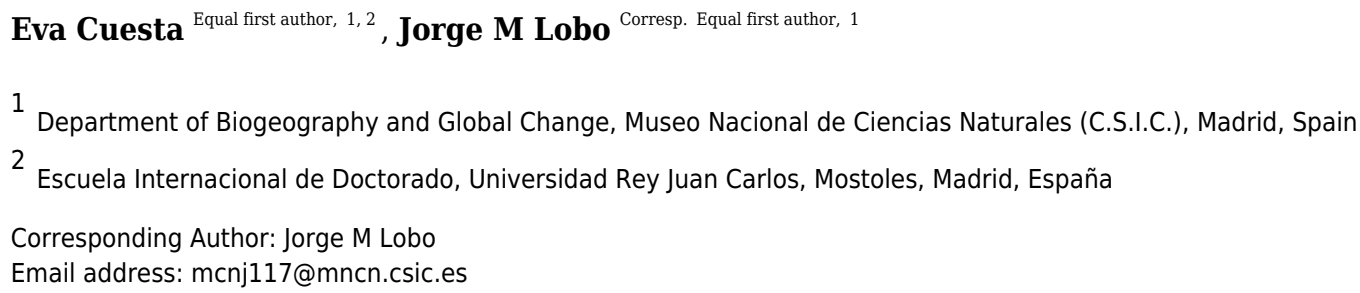

Background: The exoskeleton of an insect could be an important factor in the success of its evolutionary process. This reaches its maximum expression in beetles, which constitute the most diversified animal taxon. The involvement in the management of environmental radiation could be one of the most important functions of the exoskeleton due to the passive contributions to the thermoregulation of body temperature. We study whether the elytra of two sympatric and closely related beetle species respond differentially to the radiation of distinct wavelengths in agreement with their ecological preferences.

Methods: Onthophagus coenobita (Herbst) and O. medius (Kugelaan) occupy different habitats and environmental conditions (shaded versus unshaded from solar radiation). The potential adaptive variations to thermoregulation under these different ecological conditions were studied using the responses of their exoskeletons to radiation of different wavelengths (ultraviolet, visible and near-infrared). For these two species, the amounts of the three wavelengths that were reflected, transmitted or absorbed by the exoskeleton were measured using of a spectrophotometer. In addition, the darkness and thickness of the elytra were examined to determine whether these two features influence the management of radiation by the exoskeleton. Results: Both species differ in the management of visible and near-infrared radiation. In agreement with habitat preferences, the species inhabiting shaded conditions would allow infrared and visible radiation to penetrate the elytra more easily to heat internal body parts, while the elytra of the heliophilous species would have increased absorbance of these same types of radiation. An increase in body size (and therefore in elytron thickness) and the quantity of dark spots may serve as barriers against exogenous heat gain. However, the maintenance of between-species differences independent of the effects of these two morphological features led us to suspect that an unconsidered elytron characteristic may also be affecting these differences. Discussion: The results of the involvement of the exoskeleton 
thickness and spots in the thermoregulation of insects opens new research lines to obtain a better understanding of the function of the exoskeleton as a passive thermoregulation in Coleoptera. 
1 Visible and near-infrared radiation may be transmitted or absorbed differently by beetle

2 elytra according to habitat preference

3 Eva Cuesta $^{1,2}$, Jorge M. Lobo ${ }^{1}$

$4{ }^{1}$ Department of Biogeography and Global Change, Museo Nacional de Ciencias Naturales,

5 (C.S.I.C), Madrid, Spain.

6 2Escuela Internacional de Doctorado, Universidad Rey Juan Carlos, Móstoles (Madrid), Spain

7

8 Corresponding Author:

9 Jorge M. Lobo

10 Dpto. Biogeography and Global Change, Museo Nacional de Ciencias Naturales, (C.S.I.C),

11 Madrid, Spain. Email: Jorge.Lobo@mncn.csic.es

12

13

ORCID: JML 0000-0002-3152-4769; EC, 0000-0001-9092-5127

14

15

Running head: Elytra response to radiations

16

17 


\section{ABSTRACT}

19 Background: The exoskeleton of an insect could be an important factor in the success of its evolutionary process. This reaches its maximum expression in beetles, which constitute the most diversified animal taxon. The involvement in the management of environmental radiation could be one of the most important functions of the exoskeleton due to the passive contributions to the thermoregulation of body temperature. We study whether the elytra of two sympatric and closely related beetle species respond differentially to the radiation of distinct wavelengths in agreement with their ecological preferences.

Methods: Onthophagus coenobita (Herbst) and O. medius (Kugelaan) occupy different habitats and environmental conditions (shaded versus unshaded from solar radiation). The potential adaptive variations to thermoregulation under these different ecological conditions were studied using the responses of their exoskeletons to radiation of different wavelengths (ultraviolet, visible and near-infrared). For these two species, the amounts of the three wavelengths that were reflected, transmitted or absorbed by the exoskeleton were measured using of a spectrophotometer. In addition, the darkness and thickness of the elytra were examined to determine whether these two features influence the management of radiation by the exoskeleton.

Results: Both species differ in the management of visible and near-infrared radiation. In agreement with habitat preferences, the species inhabiting shaded conditions would allow infrared and visible radiation to penetrate the elytra more easily to heat internal body parts, while the elytra of the heliophilous species would have increased absorbance of these same types of radiation. An increase in body size (and therefore in elytron thickness) and the quantity of dark spots may serve as barriers against exogenous heat gain. However, the maintenance of between- 
40 species differences independent of the effects of these two morphological features led us to

41 suspect that an unconsidered elytron characteristic may also be affecting these differences.

42 Discussion: The results of the involvement of the exoskeleton thickness and spots in the

43 thermoregulation of insects opens new research lines to obtain a better understanding of the

44 function of the exoskeleton as a passive thermoregulation in Coleoptera.

45 Subjects Entomology, Thermal physiology, Ecology, Environmental Science.

46 Keywords Spectrophotometry, global radiation, thermoregulation, darkness, elytra thickness,

47 Onthophagus.

48 


\section{INTRODUCTION}

50 The radiation emitted by the sun can be considered the ultimate cause of the functioning of

51 biogeochemical cycles in nature, and the flux of energy created by this radiation is a decisive 52 force that conditions the behavioural, ecological, morphological, metabolic and physiological characteristics of living organisms (Hessen, 2008; Angilletta, 2009). This phenomenon is especially true for the animals, such as insects, that depend on radiation and external temperatures to warm their internal parts, thus enhancing metabolic processes and increasing evolutionary rates (Brown et al., 2004).

The development of an external skeleton that protects and supports internal body parts is an essential feature of Coleoptera, which is the most diversified animal group on Earth (Chapman, 2009) and originated during the early Permian period (Zhang et al., 2018). Among the many functions attributed to the exoskeleton of Coleoptera (Vincent \& Wegst, 2004; Gorb, 2013), some authors suggest that its structure and colour may help in controlling temperature (Mikhailov, 2001; Ishay et al., 2003; Gross et al., 2004; Clusella-Trullas et al., 2007; Davis et al., 2008; Drotz et al. 2010; Roulin, 2014; Schweiger \& Beirkuhnlein, 2016). In a series of recent studies carried out on specimens of different species belonging to the Geotrupinae and Scarabaeinae subfamilies (Coleoptera, Scarabaeoidea), the existence of "passive thermoregulation" without associated energetic costs was proposed as a consequence of the interaction of the exoskeleton with different types of electromagnetic radiation (Carrascal et al., 2017; Amore et al., 2017; Alves et al., 2018). First, heating experiments under controlled conditions on dried specimens of 13 species of Palaearctic Geotrupidae (Carrascal et al., 2017) and seven Neotropical Scarabaeinae species (Amore et al., 2017) were carried out. The evidence obtained in these studies suggested that there are interspecific differences in internal body 
72 temperatures when these specimens are exposed dorsally to simulated sunlight (Amore et al.,

73 2017), and also that these internal temperatures are lower when these specimens are exposed to

74 infrared radiation (Carrascal et al., 2017; Amore et al., 2017). In a subsequent step (Alves et al.,

75 2018), a spectrophotometric analysis was used to examine if the reflectance, transmittance and

76 absorbance of the elytra exoskeleton to different wavelengths (ultraviolet, visible and near-

77 infrared) could help to explain the obtained patterns in the internal body heat of the beetles (what

78 can be the main source of body heat?). Thus, analysing the elytra of five Neotropical

79 Scarabaeinae species of the genus Canthon a similar spectrophotometric pattern was obtained:

80 the light from shorter wavelengths is almost entirely absorbed by the elytra, while radiation from

81 longer wavelengths can mostly pass through the elytra. Consequently, this temperature increase

82 probably results from the transmittance and/or absorbance of non-infrared wavelengths by the

83 dorsal cuticle. The elytra of these species could absorb most of the highly energetic radiation

84 from the ultraviolet and visible parts of the spectrum and convert it into body heat (Alves et al.,

85 2018, Pavlovic et al., 2018). All these results suggest that the beetle exoskeleton may allow for

86 the "passive thermoregulation" of body temperatures. As the optima body temperatures of

87 individuals may tend to match the temperatures experienced in the environmental conditions

88 where they occur (Bozinovic et al., 2011; Deatherage et al., 2017), it can be hypothesized that

89 differences in the structures and colours of exoskeletons may help explain the ecological and

90 biogeographical characteristics of these organisms, as well as to understand their responses to

91 climatic changes.

92 In this study, the reflectance, transmittance and absorbance of elytra to different

93 wavelengths of electromagnetic radiation are examined in two phylogenetically close

94 Scarabaeinae species that are locally sympatric and diurnal but differ in their environmental 
95 preferences (shaded vs. open habitats). The main aim of this comparison is to verify whether

96 there might be a correspondence between the general environmental preferences of these two

97 species and their capacity to reflect, absorb or transmit radiation of different wavelengths.

98 Specifically, it is hypothesized that species inhabiting shaded conditions would be associated

99 with a higher capacity for elytra transmittance mainly in the infrared range, while heliophilous

100 species should have elytra that are able to cope with a high level of direct sunlight to minimize

101 the risk of overheating. We additionally aim to discern whether these possible interspecific

102 differences in the management of radiation can be attributed solely to differences in body mass

103 and darkness or if, alternatively, any other exoskeletal characteristic could be involved in the

104 thermoregulation mechanism.

105

106 MATERIAL \& METHODS

107 Studied species

108 Individuals of Onthophagus (Palaeonthophagus) coenobita (Herbst, 1783) and Onthophagus

109 (Palaeonthophagus) medius (Kugelann, 1792) were used in this study. The used specimens of

110 these two species were collected within the El Ventorrillo field station with the required

111 permissions provided by the Consejería de Medio Ambiente y Ordenación de Territorio of the

112 Comunidad de Madrid (approval number 10/069528.9/18). The used specimens O. medius is a

113 recently proposed taxa (Rössner et al., 2010) that is very similar to O. vacca (Linnaeus, 1767)

114 and can be accurately differentiated by using mitochondrial DNA sequences and, to a lesser

115 extent, by some subtle and overlapping morphological characters among which elytra darkness

116 stands out (Roy et al., 2016). In our case, all the studied specimens were carefully selected

117 according to non-overlapping morphological character states (Roy et al., 2016), thus the 
118 specimens were unambiguously assigned to $O$. medius. These two dung beetle species

119 (Coleoptera; Scarabaeidae) are widely distributed across the Palaearctic region. O. coenobita has 120 a geographical distribution ranging from Spain to Sweden and from Belgium to Turkmenistan.

121 Although the knowledge of the geographic distribution of $O$. medius is limited, the available data 122 suggest that $O$. vacca and $O$. medius overlap extensively in their distributions, although $O$. 123 medius is assumed to be absent in North Africa and present from Spain to Finland and Russia 124 and from Great Britain to Kazakhstan (Rössner et al., 2010; Roy et al., 2016). Different studies (Villalba et al. 2002; Roggero et al., 2017; Rössner et al., 2010; Roy et al., 2016) agree on the phylogenetic closeness of $O$. coenobita and $O$. vacca and therefore between $O$. coenobita and $O$. medius. Both species also show clear ecological differences; $O$.

128 coenobita is frequently reported to be associated with forests and shaded localities and consemes 129 human dung, corpses and mushrooms, in addition to herbivore dung (Goljan, 1953; Jessop, 1986; Lumaret, 1990; Martín-Piera \& López-Colón, 2000). Despite the lack of reliable data on the environmental preferences of $O$. medius, the available information suggests that this species has a similar ecology to $O$. vacca, which is associated with open green pastures and the consumption of cow, horse or sheep dung, but with the seasonal activity mainly focused on the warmest spring months (Rössner et al., 2010; Roy et al., 2016). A yearly non-published survey conducted during 2017 and 2018 at the "El Ventorrillo" biological station (Madrid, Spain, Lat: $40.75^{\circ}$, Long $=-4.02^{\circ}, \approx 1430 \mathrm{~m}$ a.s.1) clearly indicates that these species do not overlap environmentally but may partially coexist seasonally. While O. medius and O. coenobita do not differ in their general midday daily activity, O. coenobita shows a marked preference for woodland sites. Adults of $O$. coenobita are also active at higher air temperatures (approximately 
141 seasonal occurrence of $O$. medius (mean seasonal occurrence around 11 May) compared with $O$.

142 coenobita (mean seasonal occurrence around 7 June).

144 Body measurements and spectrophotometric analysis

145 Ten individuals of each of the two taxa preserved in $70 \%$ ethanol were randomly selected from a

146 collection of 4,502 dung beetles belonging to 53 species collected at the "El Ventorrillo"

147 biological station during 2012-2013 (collection deposited in the Museo Nacional de Ciencias

148 Naturales of Madrid). After drying, each specimen was weighed using a Tx423L Shimadzuß

149 balance with a precision of $0.001 \mathrm{~g}$. Subsequently, the left elytron of each specimen was

150 carefully removed with tweezers (Fig. 1) and mounted on black vinyl to estimate their

151 reflectance. The total area of each elytron and the proportion with dark spots were calculated

152 using ImageJ 1.52i software (Schneider et al., 2012; see Fig. 1). The area of the

153 spectrophotometer light beam falling perpendicular to the surface of the elytron was $10.89 \mathrm{~mm}^{2}$,

154 thus the beam practically covered the entire area of the elytron (see supplementary data).

155 However, when the area of the elytron was slightly smaller than the area of the light beam, the

156 obtained reflectance measurements were corrected to subtract the part of the light that fell

157 outside of the elytron by using the following equation: $\mathrm{RE}=R T-\left(R v^{*} A v\right)$, where $R E$ is the

158 elytron reflectance, $R T$ is the total obtained reflectance, $R v$ is the reflectance of the vinyl per $\mathrm{mm}^{2}$

159 for the different wavelengths, and $A v$ is the vinyl area not covered by the elytron (10.89- elytron

160 area). In the case of transmittance, such correction is unnecessary because the elytron is mounted

161 on an opaque metal plate with a hole smaller than the minor size of an elytron $\left(3.301 \mathrm{~mm}^{2}\right)$.

162 The convexity of the elytra can be considered negligible in both species. The thickness of 163 the left edge of the elytron was also measured with a Nikon Measurescope 10 monocular stereo 
164 equipped with a Nikon Digital Counter CM-6S (all measurements in mm). Each elytron was 165 measured on three different occasions by two researchers, and their data were averaged. Reflectance (R; the return of the electromagnetic radiation from the surface of the elytra)

167

168

169

170

171

172

173

174

175

176

177

178

179

180

181

182

183

184

185

186 and transmittance ( $\mathrm{T}$; the passage of the electromagnetic radiation through the elytra) of the external part of the left elytron (dorsal) were measured with a Shimadzu ${ }^{\circledR}$ UV-2600 spectrophotometer is equipped with an integrating sphere (ISR-2600Plus) that is able to measure the diffuse and specular reflectance of solid samples. In our case, the measurement conditions of the optical system were adjusted to those needed to measure diffuse reflectance due to the slightly rough characteristics of the elytral surface. Before each spectrophotometer measurement, a white plate of barium sulfate was used to correct the baseline. The obtained data covering the complete wavelength spectrum from 185 to $1400 \mathrm{~nm}$ were divided into three bands; ultraviolet (UV; 185-385 nm), visible (VIS; 390-745 nm) and near-infrared (NIR; 750-1400 nm).

Absorbance (A; the transformation of the electromagnetic radiation received by the elytra into internal energy) was estimated as $\mathrm{A}=100-(\mathrm{T}+\mathrm{R})$ (see Kinoshita, 2008). Thus, the values of T, $\mathrm{R}$ and A were averaged to obtain only one value for each of the three bands as the response variable to determine whether there was variation among elytra in response to the different wavelengths emitted by the sun. The reflectance and transmittance of the internal sides of each elytron (ventral) were also measured but in only the near-infrared range to estimate the possible capacity of the elytra to reflect or transmit body heat generated by beetles. Each measurement was repeated three times by two researchers $(2$ species x 10 individuals x 2 sides x 3 measurements $=120$ measurements for transmittance and reflectance). The three repeated measurements of transmittance and reflectance for each individual were averaged to obtain more 
187 stable data that were not dependent on the position of the elytra or the sector sampled by the 188 spectrophotometer. As the immersion of the elytra in alcohol can modify spectrophotometer 189 measurements (e.g., by eliminating cuticular hydrocarbons), the UV, VIS and NIR reflectance 190 and transmittance values of five fresh elytra of $O$. medius were estimated before and after being 191 subjected to an immersion in $96^{\circ}$ alcohol for sixteen days. Only the dorsal reflectance in the UV

192 band suggested an effect of alcohol soaking ( $t$ test $=2.81, \mathrm{df}=8 ; \mathrm{P}=0.02)$, although the 193 statistical significance of this relationship disappeared when a Bonferroni correction was applied 194 (mean UV reflectance $\pm \mathrm{SD}$; fresh elytra $=2.44 \pm 0.18$; alcohol elytra $=2.70 \pm 0.11$ ). If there was 195

a potential effect of the immersion in alcohol on elytra reflectance, we assume here that it was relatively small and similar in the two considered species.

\section{Statistical analyses}

Between-taxa differences in elytron darkness (percentage of the elytron area that was dark) and biometric variables (body mass, elytron area, and elytron thickness) were tested by means of Student's $t$-tests considering that these variables follow a normal distribution $(\mathrm{n}=10$ for each species), and the probability levels were corrected for unequal variances, if applicable. Darkness was considered in these analyses because the melanic compounds responsible for darkness are associated with the absorbance of shortwave radiation and the regulation of body heat (Pinkert \& Zeuss, 2018). Because the correlations between the three biometric variables were always positive and high (Pearson $r$ values oscillating from 0.83 to $0.96 ; \mathrm{P}<0.0001$ in all cases), elytron thickness was selected for further analyses assuming that a greater elytron thickness could negatively affect the transmittance of radiation towards the interior of the body. 
210 (internal vs. external; in the case of only NIR) in reflectance, transmittance and absorbance

211 (response variables) was examined by ANCOVAs using elytron thickness and elytron darkness

212 as covariates. In these analyses, the explanation of the additive main effects was obviated when

213 two-way interactions showed a relevant effect. Type III sums of squares were used to estimate

214 the partial effect of each explanatory variable once the effects of the other variables were

215 controlled for. The obtained standardized partial regression coefficients can be considered

216 unbiased estimates of the relative importance of predictors, even when they are highly correlated

217 (Smith et al. 2009). The effects of the species identity factor, including and excluding covariates,

218 were compared because their change in magnitude and/or sign may indicate the existence of

219 influential confounding or suppressor variables able to overestimate or underestimate the effect

220 of species identity (Legendre \& Legendre, 1998; Smith et al. 2009).

221 The use of P-values as thresholds to discriminate significant and non-significant results is

222 increasingly questioned (Halsey, 2019), which is mainly due to their inability to inform about the

223 rate of false positives (Colquhom, 2017). As a consequence, we have abandoned here the use of

224 the terms "statistically significant" and "statistically non-significant", considering P-values as

225 indicators of the strength of the evidence of the studied relationships. Thus, Bonferroni corrected

226 P-values for multiple comparisons ( 3 wavelength ranges x 3 response variable; 0.05/9=0.006)

227 were considered to identify "strong evidence" of relationships, while relationships with P-values

228 from 0.05 to 0.006 were considered "weak evidence". We checked for homoscedasticity and 229 normality in the residuals of these models. StatSoft's STATISTICA v12.0 was used for these 230 analyses 


\section{RESULTS}

\section{Biometric and colour differences}

234 The average body mass $(\mathrm{mg})$, elytron area $\left(\mathrm{mm}^{2}\right)$ and elytron thickness $(\mu \mathrm{m})$ differed between $O$. 235 coenobita and O. medius, with higher values for the latter taxon $(\mathrm{P}<0.001$, Table 1$)$. The area of 236 dark pigmentation was also lower for O. coenobita than for O. medius (Table 1).

\section{General responses of elytra to wavelength spectrum}

The average values of reflectance, transmittance and absorbance across the examined wavelength spectrum for both species and elytron sides (internal and external) are shown in Figure 2. On average, the reflectance values were lower than the absorbance and transmittance values throughout the complete wavelength spectrum, while absorbance was very high in the ultraviolet and visible wavelength ranges.

\section{The effect of elytron side}

246 The interaction between species identity and the elytron side factor was highly unlikely to

247 explain the NIR reflectance, transmittance or absorbance (probabilities higher than 0.30 in all 248 cases), indicating that the effect of the elytra side was similar in the two species (Table 2). The 249 dorsal or ventral position of the elytra did not seem to influence the NIR reflectance values, but 250 strong evidence of the influence of elytra position on transmittance and absorbance existed. The

251 ventral NIR transmittance in $O$. medius (56.4\%; adjusted means) was higher than that in $O$.

252 coenobita (45.1\%) and lower than those measured from the external side of the elytra $(52.5 \%$

253 and $37.5 \%$, respectively). Elytra NIR absorbance also seems to be influenced by the elytron side 254 factor (Table 2) as it was lower in O. medius than in O. coenobita for both the internal side (30.3 
$255 \%$ vs $45.8 \%$ ) and the dorsal side (34.7\% vs 53.5\%). Interestingly, the addition of covariates in

256 the regression analyses changed the comparative transmittance and absorbance values of the two

257 species (Table 2). O. medius had lower percentages of NIR transmittance and higher percentages

258 of NIR absorbance, both dorsally and ventrally, than $O$. coenobita when the raw data were

259 considered. However, this comparative situation was reversed when the effect of elytron

260 thickness and darkness was considered (Table 2).

261

262 The role of thickness and darkness

263 Our analyses support the existence of strong evidence of the role of elytron thickness in dorsal

264 NIR transmittance and absorbance and weak evidence of the effect of this covariate on NIR

265 reflectance and visible transmittance (Table 2). Thus, the transmittance of the NIR radiation

266 decreased and the NIR absorbance increased when the elytron thickness was higher (according to

267 the signs of the standardized coefficients); a thicker elytron thickness obstructed the penetration

268 of infrared radiation but facilitated its absorbance. The effect of thicker elytra in increasing NIR

269 reflectance and diminishing the transmission of visible radiation should be viewed with caution.

270 Darkness seems to be the most influential covariate (i.e., highest absolute values of the

271 standardized regression coefficients), showing strong evidence of being an influential variable in

272 explaining the variation in NIR and visible transmittance and absorbance (Table 2). Additionally,

273 the possibility should not be discounted that darker elytra reduce the reflectance of visible

274 radiations. Thus, darker elytra block the passage of infrared and visible radiation, but they favour

275 the absorption of these types of radiation.

276

277 Interspecific differences 
278 Our results provide strong evidence that both species differ in the dorsal transmission and

279 absorbance of NIR and visible radiations and weak evidence of interspecific differences in the

280 dorsal reflectance of these radiation types (Table 2). The elytra of $O$. medius seem to have a

281 higher capacity to prevent the passage of these two types of radiation, while the elytra of $O$.

282 coenobita would better absorb these same types of radiation when the effect of the studied

283 covariates is considered. Again, the addition of elytron thickness and elytron darkness in the

284 regression analyses reversed the comparative transmittance and absorbance values of the two 285 species (Table 2).

286

287 DISCUSSION

288 This research aims to assess whether the characteristics of the elytral exoskeleton may contribute 289 to facilitating the thermoregulation of beetles by differentially transmitting, absorbing or 290 reflecting radiation of distinct wavelengths in correspondence with the environmental

291 preferences of the species. The results obtained in this study support this assumption, although

292 more evidence will be needed to clearly discern the extent of the passive role of the beetle

293

294

295

296

297

298

299

300 exoskeleton in thermal performance. Thus, although the observed disparities can be associated with biometric and darkness differences, the thermal performance of elytra is consistent with the expectations.

Our results agree with those of previous studies (Carrascal et al., 2017; Amore et al., 2017; Alves et al., 2018) in that elytron reflectance is minimal, transmittance of infrared radiation is very high, and most of the ultraviolet and visible radiation is absorbed by the elytra. All these exoskeletal characteristics are consistent with the requirements of an ectothermic organism that spends a good deal of time in the soil and would need to obtain body heat from the surrounding

PeerJ reviewing PDF | (2019:06:38443:2:0:NEW 16 Oct 2019) 
301 infrared and visible radiation (see also Pavlovic et al., 2018). Thus, elytra seem to be highly

302 transparent to the heat coming from the sun or the environment but opaque to the most energetic

303 wavelengths capable of causing harmful effects (Beresford et al., 2013). Of course, this pattern

304 may vary in those insects exposed to the extreme temperature conditions of deserts in which

305 large parts of visible and near-infrared radiation are reflected (Shi et al., 2015). On the other

306 hand, and in agreement with previous results (Alves et al., 2018), elytral transparency to infrared

307 radiation seems to be slightly higher on the inside part of the elytron than on the outside part,

308 suggesting that the elytra can be slightly more effective at facilitating the removal of body heat in

309 these dung beetle species. In a recent paper, Pavlovic et al. (2018) demonstrated that short (1400-

$3103000 \mathrm{~nm}$ ) and mid (3000-8000) infrared wavelengths, which are mostly absorbed by atmospheric

311 gasses (Eltbaakh et al., 2011), can be used to dissipate body heat. Further studies are needed to

312 assess whether the transmittance of these infrared wavelengths is especially high from the

313 internal side of the elytra.

314 As we expected, interspecific differences in the thermal role of the exoskeleton are

315 clearly mediated by biometric and colour characteristics, as exemplified by the effects of elytron

316 thickness and area of dark pigmentation in our analyses. Elytron thickness and especially elytron

317 darkness seem to be particularly relevant in preventing the entry of NIR and visible radiation into

318 the beetle body but also in absorbing these types of radiation. The species with lighter and

319 thinner elytra, which inhabit areas with shaded conditions (O. coenobita), would allow these

320 types of radiation to penetrate the elytra more easily to heat their internal body parts. Quite the

321 contrary, the species with darker and thicker elytra (O. medius), which inhabited sunny areas,

322 appears to be better able to absorb infrared and visible radiation. These results are in agreement

323 with those of a recently published study on the thermal capacity of the elytra of the saproxylic 
324 beetle Rosalia alpina (Linnaeus, 1758) (Pavlovic et al., 2018), which inhabits the sun-exposed

325 forest along the Euro-Caucasian region. The black patches present in the elytra of this species are

326 also able to absorb visible radiation to heat its body, but the elytra also serve to quickly transmit

327 the infrared radiation to attain thermal equilibrium. may even reverse the sign of the factor representing species identity. This statistical result has been long recognised and is especially frequent when dealing with correlated predictors (Leamer, 1975). In biological and environmental data, the use of non-independent explanatory variables is the norm rather than the exception and not including a valuable predictor because it is correlated with others may imply under or overestimations of the effects of the considered predictors (Smith et al., 2009). In our case, the inclusion of covariates in the models reversed the comparative transmittance and absorbance values that could be obtained for the two considered species.

Therefore, as the species identity factor continued to be relevant when elytron darkness and thickness were considered, it could not be excluded that some additional and unknown morphostructural differences may also be relevant in explaining the detected interspecific differences in the capacity of the elytra to manage radiations. Notwithstanding the above, caution is required when determining the comparative roles of correlated features such as elytron thickness and darkness. Additional studies are thus needed to cover a broad range of species with different degrees of darkening and elytron thicknesses to better discriminate the comparative roles of biometric and colour characteristics on the thermal performance of the beetle elytral cuticle. The maintenance of the strength of the exoskeleton with the increase in body size may imply increasing thickness both allometrically and isometrically (Evans \& Sanson, 2005; Lease

$346 \&$ Wolf, 2010). Thus, an increase in the body size of dung beetles can provide extra advantages 
347 in open habitats by avoiding the internal overheating of the body under sunny conditions.

348 Similarly, the darkening of the exoskeleton could be partially considered an evolutionary

349 strategy to diminish heat transmission into the body. This supposition collides with the thermal

350 melanism hypothesis, which predicts that a darker colour may be advantageous in colder

351 environments (Kalmus, 1941; Schweiger and Beierkuhnlein, 2016, Galván et al. 2018) but could

352 explain why desert beetles are often dark (Turner \& Lombard, 1990). In our case, the darkest

353 elytra seem to make the access of infrared and visible radiations into the body more difficult,

354 also facilitating the absorbance of these types of radiation; however, the elytra do not influence

355 the management of UV radiation. As the transmittance and absorbance of solar radiation may

356 vary between the elytral parts with different colours and structures (Pavlovic et al., 2018), the

357 future use of microspectrometry will be recommended to further assess the specific role of black 358 patches in thermal balance.

359 As in the case of body size, the reduced transmittance of near-infrared radiation by the

360 dark specimens of our two considered species may be a strategy to avoid overheating under some

361 circumstances. Considering that more than $50 \%$ of the total sunlight incident energy corresponds

362 to this wavelength spectrum (Stuart-Fox et al., 2017), the management of near-infrared radiation

363 by the beetle exoskeleton should be considered. In this case, darkness can affect thermal

364 performance due to its effect on longwave radiation, which is invisible to the human eye (Stuart-

365 Fox et al., 2017). However, Pantelić et al. (2017) highlight the low capacity of melanin to absorb

366 the infrared radiation in a dusk moth species, which could indicate that the structural component

367 in which the pigment is embedded could influence this response. Additional experiments are

368 needed to better estimate whether the elytra of different beetle species differ in their capacities to 
369 manage distinct wavelengths and discern the comparative roles of body size and darkness in the 370 thermoregulation of beetles.

371

372 CONCLUSIONS

373 The main hypothesis of this research has been that the elytra of two closely related beetle species

374 will manage environmental radiation differentially in agreement with their contrasting

375 environmental preferences. Thus, the results are in line with what was expected because the 376 elytra of the species inhabiting areas under shaded conditions (O. coenobita) allow the entry of

377 infrared and visible radiation more easily, while the heliophilous species (O. medius) would 378 better absorb these same types of radiation. These differences are determined largely by the 379 thickness and darkness of the elytra, but we cannot rule out the role that other unknown factors 380 could play in these differences. Further and more comprehensive studies are needed to 381 corroborate the role of the elytral exoskeleton as a mechanism of "passive thermoregulation".

\section{ACKNOWLEDGEMENTS}

We are indebted to Luis María Carrascal for his valuable suggestions. The comments provided by three anonymous referees have improved this manuscript.

386

\section{ADDITIONAL INFORMATION AND DECLARATIONS}

\section{Funding}

This work was supported by the MINECOFEDER Project CGL2015-64489-P and the MINECOFEDER Contract BES-2016-077087 granted to the first author. 


\section{Competing Interests}

393 The authors declare there are no competing interests.

394

395

Ethical approval

396

Beetle collection was conducted with relevant permissions provided by the Comunidad de

Madrid (Dirección General de Medio Ambiente; approval number 10/069528.9/18), considering

all applicable international and national guidelines for the care and use of animals.

\section{Author Contributions}

401

402

403

404

405

406

407

408

409

410

411

412

413

Both authors participated in the design of the study, carried out spectrophotometric analyses, participated in data analysis, and drafted the manuscript.

\section{REFERENCES}

Alves VM, Hernández MI, Lobo JM. 2018. Elytra absorb ultraviolet radiation but transmit NIR radiation in Neotropical Canthon Species (Coleoptera, Scarabaeinae). Photochemistry and Photobiology 94:532-539.

Amore V, Hernández MIM, Carrascal LM, Lobo JM. 2017. Exoskeleton may influence the internal body temperatures of Neotropical dung beetles (Col. Scarabaeinae). PeerJ 5:e3349.

Angilletta MJ. 2009. Thermal Adaptation: A Theoretical and Empirical Analysis. New York: Oxford University Press. 
414 Beresford GW, Selby G, Moore JC. 2013. Lethal and sub-lethal effects of UV-B radiation 415 exposure on the collembolan Folsomia candida (Willem) in the laboratory. Pedobiologia

416

417

418

419

420

421

422

423

424

425

426

427

428

429

430

431

432

433

434

435 56:89-95.

Bozinovic F, Calosi P, Spicer JI. 2011. Physiological correlates of geographic range in animals. Annual Review of Ecology, Evolution and Systematics 42:155-179.

Brown JH, Gillooly JF, Allen AP, Savage VM, West GB. 2004. Towards a metabolic theory of ecology. Ecology 85:1771-1789.

Carrascal LM, Jiménez-Ruiz Y, Lobo JM. 2017. Beetle exoskeleton may facilitate body heat acting differentially across the electromagnetic spectrum. Physiological and Biochemical Zoology 90:338-347.

Chapman AD. 2009. Numbers of living species in Australia and the World. Australian Biodiversity Information Services, Toowoomba. Available at http://www.environment.gov.au/node/13875.

Clusella-Trullas S, Van Wyk JH, Spotila JR. 2007. Thermal melanism in ectotherms. Journal of Thermal Biology 32:235-245.

Colquhoun D. 2017. The reproducibility of research and the misinterpretation of $p$-values. Royal Society open science 4:171085.

Davis ALV, Brink DJ, Scholtz CH, Prinsloo LC, Deschodt CM. 2008. Functional implications of temperature correlated colour polymorphism in an iridescent, Scarabaeine dung beetle. Ecological Entomology 33:771-779.

Deatherage DE, Kepner JL, Bennett AF, Lenski RE, Barrick JE. 2017. Specificity of genome evolution in experimental populations of Escherichia coli evolved at different 
temperatures. Proceedings of the National Academy of Sciences of the United States of America 114:E1904-E1912

438

439

440

441

442

443

444

445

446

447

448

449

450

Doube BM. 2018. Ecosystem services provided by dung beetles in Australia. Basic and Applied Ecology 26:35-49.

Drotz MK, Brodin T, Nilsson AN. 2010. Multiple origins of elytral reticulation modifications in the West Palearctic Agabus bipustulatus complex (Coleoptera, Dytiscidae). PLoS ONE 5:e9034.

Eltbaakha YA, Ruslanb MH, Alghoulb MA, Othmanb MY, Sopianb K, Fadhelb MI. 2011. Measurement of total and spectral solar irradiance: Overview of existing research. Renewable and Sustainable Energy Reviews 15:1403-1426.

Evans AR, Sanson GD. 2005. Biomechanical properties of insects in relation to insectivory: Cuticle thickness as an indicator of insect "hardness" and "intractability". Australian Journal of Zoology 53:9-19.

Galván I, Rodríguez-Martínez S, Carrascal LM. 2018. Dark pigmentation limits thermal niche position in birds. Functional Ecology 32:1531-1540.

Goljan A. 1953. Studies on polish beetles of the Onthophagus ovatus (L.) group with some biological observations on coprophagans (Col., Scarabaeidae). Annales Musei Zoologici Polonici 25:55-81.

Gorb SN. 2013. Insect-inspired technologies: insects as a source for biomimetics. In: Vilcinskas A, ed. Insect Biotechnology. Dordrecht: Springer, 241-264

Gross J, Schmolz E, Hilker M. 2004. Thermal adaptations of the leaf beetle Chrysomela lapponica (Coleoptera: Chrysomelidae) to different climes of Central and Northern Europe. Environmental Entomology 33:799-806. 
459 Halsey LG. 2019. The reign of the p-value is over: what alternative analyses could we employ to $460 \quad$ fill the power vacuum? Biology Letters 15:20190174.

461

462

463

464

465

466

467

468

469

470

471

472

473

474

475

476

477

478

479

Hessen DO. 2008. Solar radiation and the evolution of life. In: Bjertness E, ed. Solar Radiation and Human Health. Oslo: The Norwegian Academy of Science and Letters, 123-134.

Ishay JS, Pertsis V, Rave E, Goren A, Bergman DJ. 2003. Natural thermoelectric heat pump in social wasps. Physical Review Letters 90:218102.

Jessop L. 1986. Dung Beetles and Chafers. Coleoptera: Scarabaeoidea (New Edition). Handbooks for the Identification of British Insects 5:1-53.

Kalmus H. 1941. Physiology and ecology of cuticle colour in insects. Nature 148:428-431.

Kinoshita S. 2008. Structural colors in the realm of nature. Singapore: World Scientific.

Leamer EE. 1975. A Result on the sign of restricted least-squares estimates. Journal of Econometrics 3: 387-390.

Lease HM, Wolf BO. 2010. Exoskeletal chitin scales isometrically with body size in terrestrial insects. Journal of Morphology 271:759-768.

Legendre P, Legendre L. 1998. Numerical Ecology. Elsevier,Amsterdam.

Lumaret JP. 1990. Atlas des Coleopteres Scarabeides Laparosticti de France. Paris: Museum National d'Histoire Naturelle.

Martín-Piera F, López-Colón JI. 2000. Coleoptera Scarabaeoidea I. Fauna Ibérica (Vol. 14). Madrid: CSIC Press.

Mikhailov YE. 2001. Significance of color polymorphism in mountain populations of abundant leaf beetles (Coleoptera, Chrysomelidae). Pirineos 156:57-68 
480

481

482

483

484

485

486

487

488

489

490

491

492

493

494

495

496

497

498

499

500

Pavlović D, Vasiljević D, Salatić B, Lazović V, Dikić G, Tomić L, Ćurčić S, Milovanović P, Todorović D, Pantelić DV. 2018. Photonic structures improve radiative heat exchange of Rosalia alpina (Coleoptera: Cerambycidae). Journal of Thermal Biology 76:126-138.

Pinkert S, Zeuss D. 2018. Thermal biology: Melanin-based energy harvesting across the tree of life. Current Biology 28:871-894.

Roggero A, Barbero E, Palestrini C. 2017. Revised classification and phylogeny of an Afrotropical species group based on molecular and morphological data, with the description of a new genus (Coleoptera: Scarabaeidae: Onthophagini). Organisms Diversity \& Evolution 17:181-198.

Rössner E, Schönfeld J, Ahrens D. 2010. Onthophagus (Palaeonthophagus) medius (Kugelann, 1792) — a good western palaearctic species in the Onthophagus vacca complex (Coleoptera: Scarabaeidae: Scarabaeinae: Onthophagini). Zootaxa 2629:1-28.

Roulin A. 2014. Melanin-based colour polymorphism responding to climate change. Global Change Biology 20:3344-3350

Roy L, Bon M-C, Cesarini C, Serin J, Bonato O. 2016. Pinpointing the level of isolation between two cryptic species sharing the same microhabitat: a case study with a scarabaeid species complex. Zoologica Scripta 45:407-420.

Schneider CA, Rasband WS, Eliceiri KW. 2012. NIH Image to ImageJ: 25 years of image analysis. Nature Methods 9:671-675.

Schweiger AH, Beierkuhnlein C. 2016. Size dependency in colour patterns of Western Palearctic carabids. Ecography 39:846-857. 
501 Shi NN, Tsai CC, Camino F, Bernard GD, Yu N, Wehner R. 2015. Keeping cool: Enhanced $502 \quad$ optical reflection and radiative heat dissipation in Saharan silver ants. Science 349:298$503 \quad 301$.

504 Stuart-Fox D, Newton E, Clusella-Trullas S. 2017. Thermal consequences of colour and near505 infrared reflectance. Philosophical Transactions of the Royal Society B: Biological $506 \quad$ Sciences 372:20160345.

507 Smith AC, Koper N, Francis CM, Fahring L. 2009. Confronting collinearity: comparing 508 509 methods for disentangling the effects of habitat loss and fragmentation. Landscape Ecology 24:1271-1285

Turner JS, Lombard AT. 1990. Body color and body temperature in white and black Namib desert beetles. Journal of Arid Environments 19:303-315. Journal of Molecular Evolution 55:116-126. Structure \& Development 33:187-199. Communications 9: 205. 


\section{Table $\mathbf{1}$ (on next page)}

Morphometric values of Onthophagus coenobita and $O$. medius.

Mean and standard deviation (sd) of the considered darkness and biometrical variables among $O$. coenobita $(\mathrm{N}=10)$ and $O$. medius $(\mathrm{N}=10)$ specimens. Student's $t$-tests, corrected for unequal variances, were used to establish statistical differences in these parameters between the two species. 
1 Table 1:

2 Morphometric values of Onthophagus coenobita and $\boldsymbol{O}$. medius. Mean and standard deviation

3 (sd) of the considered darkness and biometrical variables among $O$. coenobita $(\mathrm{N}=10)$ and $O$.

4 medius $(\mathrm{N}=10)$ specimens. Student's $t$-tests, corrected for unequal variances, were used to 5 establish statistical differences in these parameters between the two species.

\begin{tabular}{lcccccc} 
& \multicolumn{7}{c}{ O. coenobita } & \multicolumn{2}{c}{ O. medius } \\
& mean & sd & mean & sd & $t$ & P \\
\cline { 2 - 7 } Body mass $(\mathrm{mg})$ & 52.70 & 12.68 & 104.90 & 33.43 & 4.62 & $<0.001$ \\
Elytral area $\left(\mathrm{mm}^{2}\right)$ & 8.79 & 1.35 & 13.11 & 1.94 & 5.78 & $<0.001$ \\
Elytral thickness $(\mu \mathrm{m})$ & 82.10 & 7.52 & 100.10 & 9.90 & 4.58 & $<0.001$ \\
\% Darkness & 3.68 & 1.83 & 24.59 & 5.20 & 12.00 & $<0.001$
\end{tabular}

6 


\section{Table 2 (on next page)}

ANCOVAs results using species identity (Onthophagus coenobita and 0 . medius) and elytron side as factors and elytron thickness and elytron darkness as covariates to estimate its effects on reflectance $(R)$, transmittance $(T)$ and absorbance $(A)$

$\beta$ are the standardized regression coefficients obtained in the regression analyses representing the comparative magnitude and sign of the predictor variables. Results including elytron side and the interaction species $x$ side are only estimated in the case of NIR ( $\beta$ is negative if the average of the internal side is higher than that for the external side). In the case of the species factor $\beta$ is negative if the average of $O$. medius is higher than that for O. coenobita. Those relationships showing P-values equal or lower than a Bonferroni corrected P-value for multiple comparisons $(0.05 / 9=0.006)$ are considered as "strong evidences" (in underlined bold), while relationships with P-values from 0.05 to 0.006 are considered as "weak evidences" (in bold). The two first columns represent average dorsal R, T or A percentages for each species taking into account raw data (first figure) and adjusted means taking into account the effect of the covariates estimated considering that the effect of the covariates is zero in the two species (second figure). 
1 Table 2:

2 ANCOVAs results using species identity (Onthophagus coenobita and $O$. medius) and

3 elytron side as factors and elytron thickness and elytron darkness as covariates to estimate

4 its effects on reflectance ( $R)$, transmittance ( $T$ ) and absorbance (A) for three different

5 wavelength ranges (NIR = near infrared from 750 to $1400 \mathrm{~nm}$; VIS= visible from 390 to 745

$6 \mathrm{~nm}$; and $\mathrm{UV}=$ ultraviolet from 185 to $385 \mathrm{~nm}) . \beta$ are the standardized regression coefficients

7 obtained in the regression analyses representing the comparative magnitude and sign of the

8 predictor variables. Results including elytron side and the interaction species $\mathrm{x}$ side are only

9 estimated in the case of $\operatorname{NIR}(\beta$ is negative if the average of the internal side is higher than that

10 for the external side). In the case of the species factor $\beta$ is negative if the average of $O$. medius is

11 higher than that for $O$. coenobita. Those relationships showing P-values equal or lower than a

12 Bonferroni corrected P-value for multiple comparisons $(0.05 / 9=0.006)$ are considered as "strong

13 evidences" (in underlined bold), while relationships with P-values from 0.05 to 0.006 are

14 considered as "weak evidences" (in bold). The two first columns represent average dorsal R, T or

15 A percentages for each species taking into account raw data (first figure) and adjusted means

16 taking into account the effect of the covariates estimated considering that the effect of the

17 covariates is zero in the two species (second figure). 


\begin{tabular}{|c|c|c|c|c|c|c|c|c|}
\hline & $\begin{array}{c}\text { O.coenobit } \\
a\end{array}$ & $\begin{array}{c}O . \\
\text { medius }\end{array}$ & Species & Elytron thickness & Elytron darkness & Elytron side & Species $\mathrm{x}$ side & $R^{2}$ \\
\hline $\begin{array}{l}\mathrm{R}- \\
\text { NIR }\end{array}$ & $10.0 / 9.0$ & $11.8 / 12.8$ & 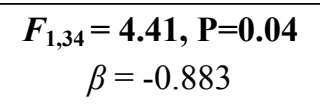 & $\begin{array}{c}\boldsymbol{F}_{\mathbf{1}, \mathbf{3 4}}=\mathbf{5 . 4 2}, \mathbf{P}=\mathbf{0 . 0 3} \\
\beta=0.400\end{array}$ & $\begin{array}{c}F_{1,34}=2.95, \mathrm{P}=0.10 \\
\beta=-0.722\end{array}$ & $\begin{array}{c}F_{1,34}=0.21, \mathrm{P}=0.65 \\
\beta=-0.063\end{array}$ & $\begin{array}{c}F_{1,34}=0.13 \\
\beta=0.0 \overline{4} 9\end{array}$ & $34.12 \%$ \\
\hline $\begin{array}{l}\text { T- } \\
\text { NIR }\end{array}$ & $46.4 / 37.5$ & $43.5 / 52.5$ & $\begin{array}{c}\underline{\boldsymbol{F}}_{1,34}=\frac{\mathbf{1 2 . 8 5}}{\hat{m}} \\
\beta=-0.973\end{array}$ & $\begin{aligned} \underline{\boldsymbol{F}}_{1,34} & =\frac{\mathbf{2 9 . 7 6}}{\sim} \\
\bar{\beta}=-0.605 & \end{aligned}$ & $\begin{array}{c}\underline{\boldsymbol{F}}_{\mathbf{1 , 3 4}}=\frac{\mathbf{1 4 . 5 3}}{\sim} \\
\bar{\beta}=-1.031\end{array}$ & $\begin{array}{c}\underline{\boldsymbol{F}}_{\mathbf{1 . 3 4}}=\frac{\mathbf{2 2 . 7 4}}{\sim} \\
\bar{\beta}=-0.428\end{array}$ & $\begin{array}{c}F_{1,34}=2.31 \\
\beta=-0.136\end{array}$ & $72.61 \%$ \\
\hline $\begin{array}{l}\text { A- } \\
\text { NIR }\end{array}$ & $43.6 / 53.5$ & $44.7 / 34.7$ & $\begin{array}{c}\underline{F}_{\mathbf{1 , 3 4}}=\mathbf{1 5 . 2 3}, \\
\bar{\beta}=1.261\end{array}$ & $\frac{\boldsymbol{F}_{1,34}=\mathbf{1 2 . 3 6}}{\hat{\beta}=0.465}$ & $\frac{\boldsymbol{F}_{\mathbf{1 , 3 4}}=\mathbf{1 5 . 3 3 ,}}{\bar{\beta}=1.2 \hat{\mathrm{n}} \dot{4}}$ & $\begin{aligned} \underline{F}_{1.34} & =\mathbf{1 7 . 3 5}, \\
\bar{\beta} & =0.44 \hat{n}\end{aligned}$ & $\begin{array}{c}F_{1,34}=1.23 \\
\beta=0.118\end{array}$ & $61.14 \%$ \\
\hline $\begin{array}{l}\text { R- } \\
\text { VIS }\end{array}$ & $4.2 / 3.1$ & $4.7 / 5.8$ & $\begin{array}{c}\boldsymbol{F}_{1,16}=\mathbf{6 . 1 0}, \mathbf{P}=\mathbf{0 . 0 3} \\
\beta=-1.519\end{array}$ & $\begin{array}{c}F_{1,16}=0.99, \mathrm{P}=0.33 \\
\beta=0.250\end{array}$ & $\begin{array}{c}\boldsymbol{F}_{1,16}=\mathbf{5 . 6 3}, \mathbf{P}=\mathbf{0 . 0 3} \\
\beta=-1.458\end{array}$ & & & $33.78 \%$ \\
\hline T-VIS & $17.0 / 9.0$ & $14.0 / 22.0$ & $\begin{array}{c}\boldsymbol{F}_{1,16}=\mathbf{9 . 6 9}, \mathbf{P}=\mathbf{0 . 0 0 7} \\
\beta=-1.470\end{array}$ & $\begin{array}{c}\boldsymbol{F}_{1,16}=\mathbf{5 . 8 5}, \mathbf{P}=\mathbf{0 . 0 3} \\
\beta=-0.467\end{array}$ & $\begin{aligned} & \underline{F}_{1,16}=\mathbf{1 2 . 0 1} \\
& \hat{\beta}=-1.636\end{aligned}$ & & & $60.94 \%$ \\
\hline $\begin{array}{l}\text { A- } \\
\text { VIS }\end{array}$ & $78.8 / 87.9$ & $81.3 / 72.2$ & $\underline{\boldsymbol{F}}_{\frac{1,16}{\hat{\beta}}}=\frac{\mathbf{1 1 . 9 3}}{\hat{\beta}=1.674}$ & $\begin{array}{c}F_{1,16}=3.92, \mathrm{P}=0.06 \\
\beta=0.393\end{array}$ & $\frac{\underline{F}_{1,16}=\frac{\mathbf{1 4 . 1 0}}{\hat{\beta}}=1.819}{\hat{\beta}}$ & & & $58.85 \%$ \\
\hline R-UV & $1.8 / 1.9$ & $2.5 / 2.5$ & $\begin{array}{c}F_{1,16}=1.63, \mathrm{P}=0.22 \\
\beta=-0.611\end{array}$ & $\begin{array}{c}F_{1,16}=0.51, \mathrm{P}=0.49 \\
\beta=0.139\end{array}$ & $\begin{array}{c}F_{1,16}=0.03, \mathrm{P}=0.87 \\
\beta=0.078\end{array}$ & & & $59.94 \%$ \\
\hline T-UV & $1.1 / 0.4$ & $2.2 / 2.9$ & $\begin{array}{c}F_{1,16}=0.83, \mathrm{P}=0.38 \\
\beta=-0.652\end{array}$ & $\begin{array}{c}F_{1,16}=0.17, \mathrm{P}=0.68 \\
\beta=-0.122\end{array}$ & $\begin{array}{c}F_{1,16}=0.22, \mathrm{P}=0.66 \\
\beta=-0.324\end{array}$ & & & $10.02 \%$ \\
\hline A-UV & $97.0 / 97.7$ & $95.3 / 94.7$ & $\begin{array}{c}F_{l, 16}=1.24, \mathrm{P}=0.28 \\
\beta=0.752\end{array}$ & $\begin{array}{c}F_{1,16}=0.09, \mathrm{P}=0.76 \\
\beta=0.085\end{array}$ & $\begin{array}{c}F_{l, 16}=0.18, \mathrm{P}=0.67 \\
\beta=0.290\end{array}$ & & & $20.01 \%$ \\
\hline
\end{tabular}


Figure 1

Habitus and elytra of Onthophagus coenobita (A) and O. medius (B).

The left elytra was removed, showing coloured external and pale internal sides.

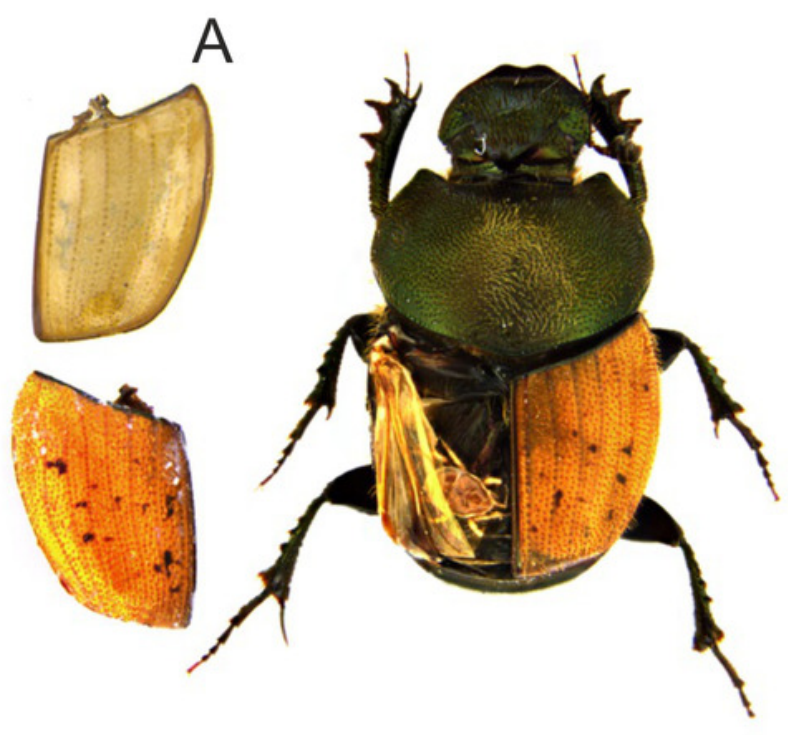

$5 \mathrm{~mm}$

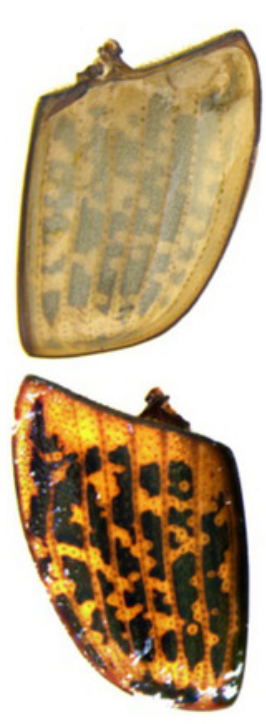

$B$

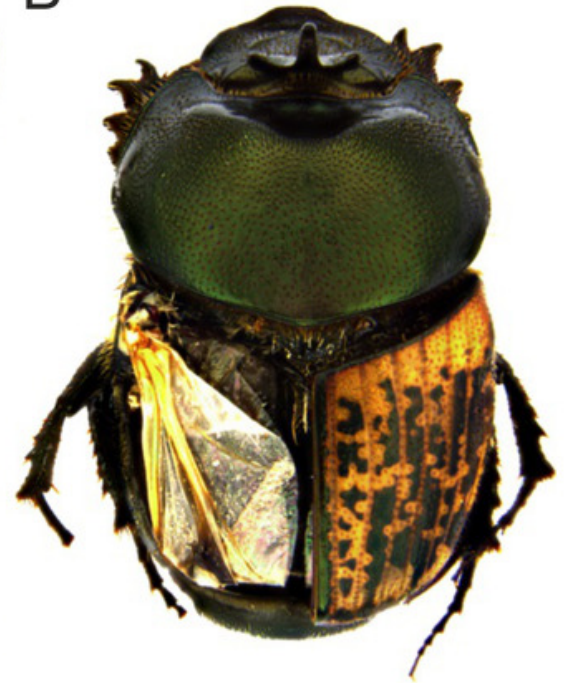

$5 \mathrm{~mm}$ 


\section{Figure 2}

Spectrophotometric graphs.

Mean absorbance (ABS), transmittance (TRA) and reflectance (REF) from 185 to $1400 \mathrm{~nm}$ of ten individuals of $O$. coenobita $(A, B)$ and $O$. medius $(C, D)$, both for the external $(A, C)$ and the internal sides of the elytra $(B, D)$. The comparison between the two species was facilitated by including a thin broken line representing the transmittance pattern of $O$. medius in the plot of $O$. coenobita. The peak observed at $830 \mathrm{~nm}$ is due to the automatic detector change wavelength (the photomultiplier and the InGaAs detector).

A

O. coenobita, external side

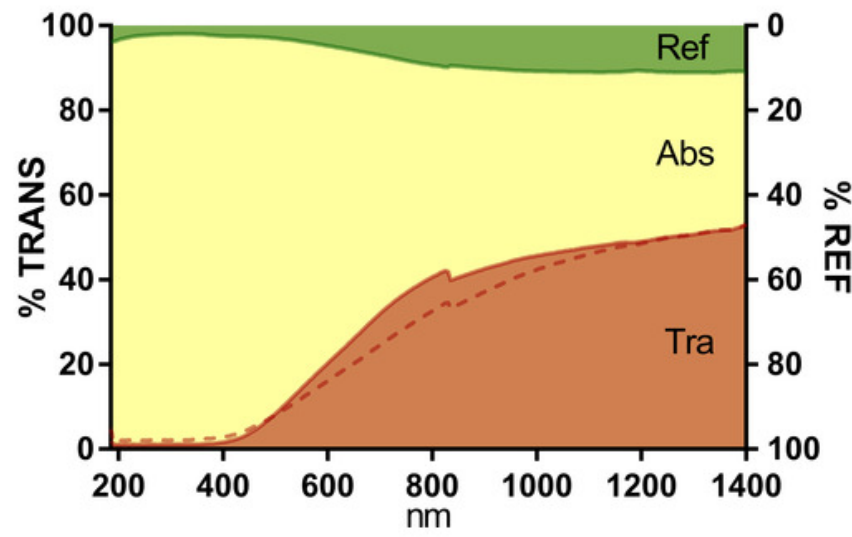

O. medius, external side

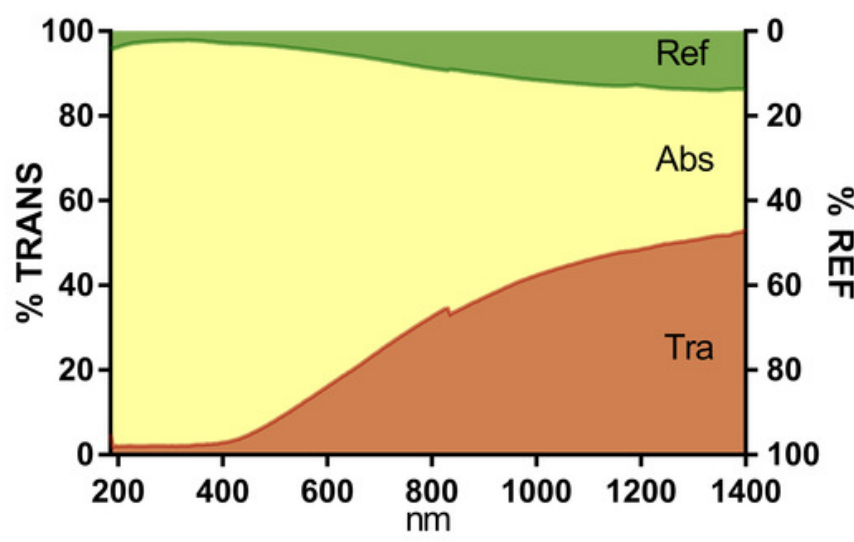

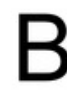

O. coenobita, internal side

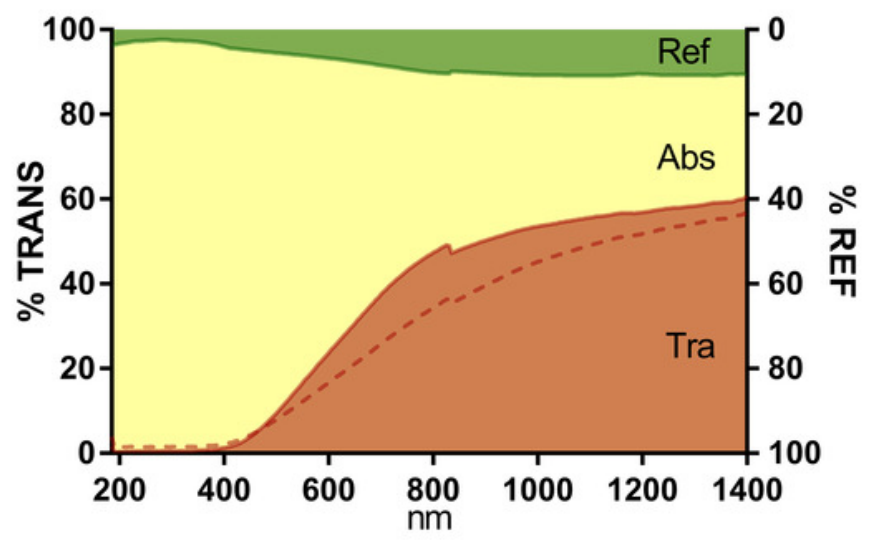

$D$ o. medius, internal side

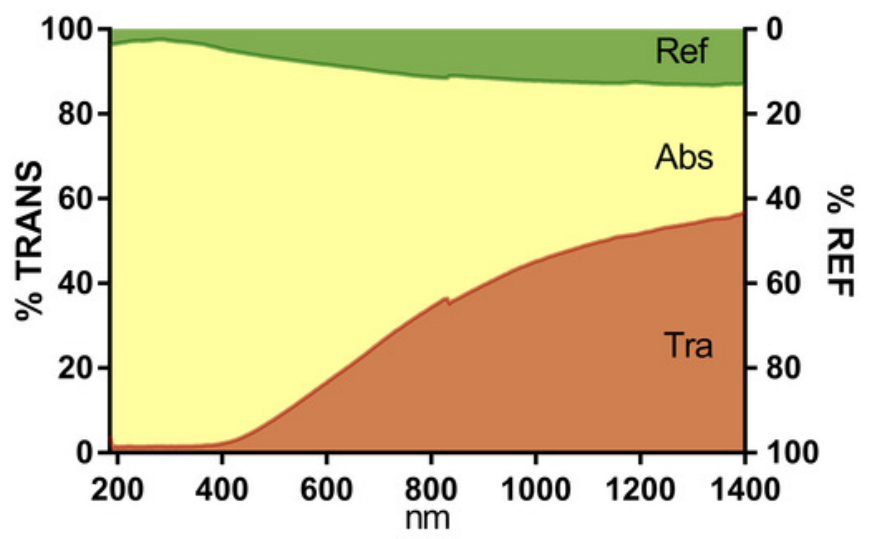

